\title{
Nutritional Problems and Solutions for the Modern Health Epidemic
}

\author{
Jing X. Kang
}

Massachusetts General Hospital and Harvard Medical School, Boston, Mass., USA

Over the last few decades, the incidence and prevalence of chronic diseases - particularly obesity, diabetes, and cancer - has continued to rise, affecting hundreds of millions of people around the world. Since the 1980s, for instance, the incidence of diabetes in the United States has increased by 133\% [1], while worldwide obesity has more than doubled [2]. The growing threat of chronic diseases to global health calls for more effective measures to stop the health epidemic. To this end, the root causes must be addressed. Although many factors may be involved, ranging from genetics to lifestyle, chronic disease is very much a matter of disordered metabolism, which can be profoundly affected by the nutrients we eat. The association of chronic disease with nutrient metabolism points to the possibility of certain dietary or nutritional factors as underlying causes of today's health epidemic. In my opinion, any dietary factors considered as the causes should satisfy two key criteria: (1) a shift (increase or decrease) in the intake of the particular nutrients correlates with an increase in the prevalence of chronic disease, and (2) the nutrients play a determining role in the key pathological processes, such as chronic low-grade inflammation and increased lipogenesis, that are the common mechanisms for chronic diseases.

The Bellagio Report by Simopoulos et al. [3] and the latest update published in this issue [4] highlight two potential causes that may account for the upward trend of chronic disease incidence and prevalence during the last century. The first cause may be an increase in the dietary intake of omega- 6 fatty acids and a decrease in the dietary intake of omega- 3 fatty acids. These changes in omega- 6 and omega- 3 fatty acid intake have dramatically shifted the omega-6/omega-3 fatty acid ratio in both our diet and bodies from the human evolutionary ratio of $\sim 1: 1$ to the modern dietary ratio ranging from 10:1 to 50:1, due to the industrialization of agriculture, processed foods, grain-fed livestock, and the increased consumption of vegetable oils [5]. This shift in the omega-6/omega-3 ratio has profound implications on human health, as these two classes of essential fatty acids are key components of cellular 
membrane structure and function. They compete for the same enzymes for biosynthesis and metabolism and have opposing effects on many physiological and pathological processes including inflammation, oxidative stress, lipogenesis, gut microbiota, and energy metabolism - which underlie the development of many chronic diseases. As outlined in the Bellagio Report, a growing body of evidence from molecular and animal to human levels shows that increasing the omega-6/omega-3 ratio can increase the risk of chronic disease, while balancing or reducing the omega-6/omega-3 ratio can have protective effects and reduce the incidence of chronic disease $[3,6]$. Although further research is warranted, the role of the omega- $6 /$ omega-3 fatty acid ratio in chronic disease cannot be underestimated.

The second cause for the increased incidence of chronic disease may be an increase in the dietary intake of fructose. The widespread use of high-fructose corn syrup in the Western diet and its prevalence in sugary beverages such as soda can significantly impact our energy metabolism. Studies have shown that fructose bypasses many of the steps of cell-mediated, energy-dependent glycolysis and induces uncontrolled lipogenesis, leading to obesity and fatty liver disease [7]. In fact, recent research has shown that sugary drinks are the top calorie source in teens' diets [8]. People who regularly consume sugary drinks (1-2 cans a day or more) have a $26 \%$ greater risk of developing type 2 diabetes than those who rarely have such drinks [9]. It is especially important to note that the combination of an unbalanced omega-6/ omega-3 fatty acid ratio and an increase in fructose intake has shown synergistic effects on impaired cognitive performance and the development of metabolic syndrome [10]. The harmful impact of an increased fructose intake will gain more recognition as further research evidence emerges. It is therefore conceivable that the omega-6/omega-3 imbalance and the increased fructose intake are two major contributors to the modern prevalence of chronic disease.

These two phenomena also emphasize a critical point: not all calories are the same. Although a given amount of omega- 6 and omega- 3 fats can generate the same number of calories, their respective outcomes on physiological and pathological conditions are markedly different. Similarly, fructose has the same caloric energy as glucose but will differentially impact health conditions. This reality suggests that the current calorie-based dietary recommendations must be revised to account for the calorie source. Furthermore, as pointed out in the Bellagio Report, another critical point that requires our attention is that genetic variation can significantly affect metabolism and individual response to diet. For example, studies have shown that the Western diet more adversely affects individuals with FADS1 and FADS2 variants by further increasing omega- 6 arachidonic acid production, leading to cardiovascular disease and diabetes [11]. This, along with the issue of calorie source, highlights the importance of personalized nutrition in the fight against chronic disease.

In order to stop the modern health epidemic, efforts must be devoted to balancing the omega-6/omega-3 ratio and reducing fructose consumption. It is my opinion that comprehensive measures should be taken from food production to food choice, from government agencies to consumers. A major issue is that current practices in food production and processing, which largely determine the availability of nutrients to consumers, have produced many unhealthy foods that require us to make dietary adjustments. Instead, agriculture and food production should be conducted based on scientifically sound nutritional guidelines. It is also necessary to get members from all levels of society involved, including policy makers, researchers, medical professionals, and educators, in order to fully implement healthy practices. To this end, an international network of centers for genetics, nutrition and fitness for health was recently established based on the Bellagio Report to promote international research collaboration, policy making based on scientific evidence, and public awareness and education. Through these efforts, I hope that we can achieve the goal of 'Healthy Agriculture, Healthy Nutrition, Healthy People'. 


\section{References}

1 Centers for Disease Control and Prevention: Crude and age-adjusted incidence of diagnosed diabetes per 1,000 population aged 18-79 years, United States, 1980-2011. 2014. http://www.cdc.gov/diabetes/ statistics/incidence/fig2.htm/ (accessed January 15, 2015).

2 World Health Organization: Obesity and overweight. http://www.who.int/mediacentre/factsheets/fs311/ en/ (accessed January 15, 2015).

3 Simopoulos AP, Bourne PG, Faergeman 0: Bellagio report on healthy agriculture, healthy nutrition, healthy people. J Nutrigenet Nutrigenomics 2013;6:34-42.

4 Simopoulos AP: The impact of the Bellagio Report on Healthy Agriculture, Healthy Nutrition, Healthy People: scientific and policy aspects. J Nutrigenet Nutrigenomics 2014;7:189-209.

5 Blasbalg TL, Hibbeln JR, Ramsden CE, Majchrzak SF, Rawlings RR: Changes in consumption of omega-3 and omega-6 fatty acids in the United States during the 20th century. Am J Clin Nutr 2011;93:950-962.

6 Kang JX: Balance of omega-6/omega-3 essential fatty acids is important for health. The evidence from gene transfer studies. World Rev Nutr Diet 2005;95:93-102.

7 Dekker MJ, Su Q, Baker C, Rutledge AC, Adeli K: Fructose: a highly lipogenic nutrient implicated in insulin resistance, hepatic steatosis, and the metabolic syndrome. Am J Physiol Endocrinol Metab 2010;299:E685-E694.

8 National Cancer Institute: Risk factor monitoring and methods. Mean intake of energy and mean contribution (kcal) of various foods among US population, by age, NHANES 2005-06. http://riskfactor.cancer.gov/diet/ foodsources/ (accessed June 21, 2012).

9 Malik VS, Popkin BM, Bray GA, Despres JP, Willett WC, Hu FB: Sugar-sweetened beverages and risk of metabolic syndrome and type 2 diabetes: a meta-analysis. Diabetes Care 2010;33:2477-2483.

10 Simopoulos AP: Dietary omega-3 fatty acid deficiency and high fructose intake in the development of metabolic syndrome, brain metabolic abnormalities, and non-alcoholic fatty liver disease. Nutrients 2013;5:29012923.

11 Martinelli N, Girelli D,Malerba G, Guarini P, Illig T, Trabetti E, SandriM, Friso S, Pizzolo F, Schaeffer L, Heinrich J, Pignatti PF, Corrocher R, Olivieri O: FADS genotypes and desaturase activity estimated by the ratio of arachidonic acid to linoleic acid are associated with inflammation and coronary artery disease. Am J Clin Nutr 2008; 88:941-949. 